\title{
A new constitutive model for prediction of impact rates response of polypropylene
}

\author{
M.I. Okereke ${ }^{1,2}$, C.H. Le ${ }^{2}$, and C.P. Buckley ${ }^{1}$ \\ ${ }^{1}$ Department of Engineering Science, University of Oxford, Oxford, OX1 3PJ, UK \\ ${ }^{2}$ Department of Engineering Systems, University of Greenwich, Kent, ME4 4TB, UK
}

\begin{abstract}
This paper proposes a new constitutive model for predicting the impact rates response of polypropylene. Impact rates, as used here, refer to strain rates greater than 1000 1/s. The model is a physically based, three-dimensional constitutive model which incorporates the contributions of the amorphous, crystalline, pseudo-amorphous and entanglement networks to the constitutive response of polypropylene. The model mathematics is based on the well-known Glass-Rubber model originally developed for glassy polymers but the arguments have herein been extended to semi-crystalline polymers. In order to predict the impact rates behaviour of polypropylene, the model exploits the well-known framework of multiple processes yielding of polymers. This work argues that two dominant viscoelastic relaxation processes - the alpha- and beta-processes - can be associated with the yield responses of polypropylene observed at low-rate-dominant and impact-rates dominant loading regimes. Compression test data on polypropylene have been used to validate the model. The study has found that the model predicts quite well the experimentally observed nonlinear rate-dependent impact response of polypropylene.
\end{abstract}

\section{Introduction}

The constitutive response of semi crystalline polymers and in particular polypropylene is complicated by the multiphase morphology of this class of polymers. Several researchers have been working on robust holistic models for semi-crystalline polymers. Hong and co-workers [1] proposed what they described as a three-component model for semi-crystalline polymers. The model was developed strictly for tensile deformation of semi-crystalline polymers and validated against tests carried out on polyethylene. The authors postulated that the cumulative tensile deformation response of a given semi-crystalline polymer can be divided into three constituent quasi-static stresses arising from the microstructure of the material. i.e. (i) a relaxing stress, $\sigma_{\mathrm{r}}-$ the stress that arises from the viscous forces within the sample, due to the separable interaction of the crystalline and amorphous phases; (ii) a crystal block stress, $\sigma_{\mathrm{c}}$ transmitted through the crystal blocks of the crystalline phase; and (iii) a network stress, $\sigma_{\mathrm{n}}$ which captures the global amorphous phase network response of a chosen semi-crystalline polymer. In their model, the authors assumed an ideal rubbery elasticity relation with material response characterized by a shear modulus, G. Again, Arruda and Wang [2] developed a three-dimensional model which was based on representative microstructural consistency, three-dimensional large deformation and strain rate dependence, while accurately replicating some of the unique experimental observations of semi-crystalline polymers. The test material used for model validation was thermoplastic olefin (TPO) with comparisons built from understandings of polypropylene deformation response. Sweeney and co-workers [3] proposed a constitutive model for prediction of large deformation of polypropylene under multiaxial loading and processing conditions. The model was developed on assumption of a mechanical analogue consisting of two parallel 'arms' - one arm consisting of a single Eyring process in series with an Edwards-Vilgis network while a second arm was entirely an Edwards-Vilgis network. The model was validated using biaxial experiments conducted at high temperature $\left(135^{\circ} \mathrm{C}\right)$. The model predicts the essential feature of material behaviour such as: large deformations, strain rate dependence and yielding.

All the above models have been developed to predict low-rate (quasi-static) response. The constitutive modelling of impact rates response of semicrystalline polymers remains in its infancy. Some of the most recent attempts have followed a phenomenological approach [4-6]. The challenge for impact rates response stem from the coupled interaction of inertial effects and rate-dependent response. The former can be determined by adequate explicit or implicit wave propagation algorithms while the later is modelled using different nonlinear rate-dependent constitutive models [7]. The later presents a real challenge when dealing with polymers which are known for their nonlinear viscoelastic behaviour and show multiple relaxation processes across a wide range of strain rates. Mulliken and Boyce recently observed and modelled this for a glassy polymer - a monophasic system [8]. However, for semicrystalline polymers the constitutive response is complicated by the multiphasic morphology. The research question that needs to be addressed is the significance of such morphology in the impact rates response of semicrystalline polymers.

An approach towards understanding the dependence on morphology at impact rates would be phenomenological i.e. observing experimentally the constitutive response of a given polymer across a wide range of strain rates. Many years ago, Chou et al. [9] showed that the dependence of yield stress on logarithm of strain rate for polypropylene follows a non-linear or bilinear [10] function. This finding has been confirmed in recent times independently by Gomez Del Rio et al. [11] and Okereke et al. [12]. Ree and Eyring [13] argued that such nonlinearity can be attributed to the coupled interaction of two activation 


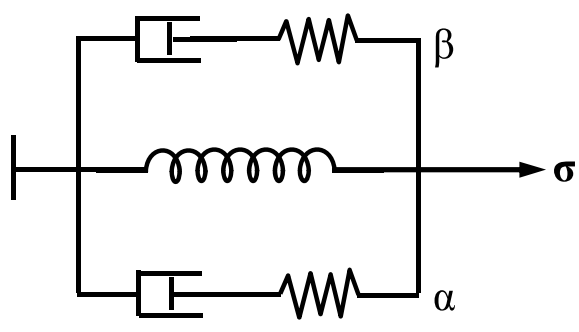

Fig. 1. A three-arm one-dimensional mechanical analogue of the two process model showing contributions of two viscoelastic processes (alpha- and beta-processes) and entanglement network.

processes each dominating at either low-rate (hightemperature) or impact rates (low-temperature) regimes. The link between viscoelastic relaxation processes and flow stress of a polymeric material was made by Bauwens [14]. Therefore, this paper exploits those arguments in developing constitutive models for polypropylene capable of predicting the constitutive behaviour at both low and impact rates.

\section{The constitutive model}

\subsection{The three-arm mechanical analogue}

A probable one-dimensional mechanical analogue for modelling deformation of such semi-crystalline polymers is shown in Fig 1. It consists of two viscoelastic arms (springdashpot arrangement) for the alpha- and beta-relaxations as well as a rubbery network spring.

\subsection{Model formulation}

The stress tensor, $\sigma$ associated with the deformation is a cumulative parallel response of the three parts of the mechanical analogue shown in Fig. 1. The proposed constitutive model consists of a set of simultaneous equations whose solution gives the Cauchy stress, $\sigma$ in terms of the deformation gradient, $\mathbf{F}$ and its rate of change, D. For the purpose of this paper, let us define the volume ratio, $J$ and mean stress: $\sigma_{\mathrm{m}}=K \ln J$ where $J=\operatorname{det} \mathbf{F}$. In this definition of mean stress, the hydrostatic response is assumed to be time independent. To develop the expression for the deviatoric component of Cauchy stress, $\mathbf{S}$ the arguments of the Glass-Rubber model for amorphous polymers [15-18] have been adopted here. The authors postulated that the total stress experienced by a given polymer results from two key energy components: (i) the bond-stretching deviatoric stress component, arising from perturbation of inter-atomic potentials; and (ii) the conformational deviatoric stress component, arising from entropy-elastic perturbation of molecular conformations.

In dealing with the semi-crystalline polymers (e.g. PE, $\mathrm{PP}, \mathrm{POM}$ ) that have an alpha-crystalline relaxation, the constitutive mathematics of the Glass-Rubber model is here extended to a biphasic two-process polymer system.

With respect to the three arm rheological model shown in Fig. 1, the deformation of semicrystalline polymers is a cumulative parallel response of the amorphous, crystalline (including a constrained 'pseudo-amorphous' zone) as well as entanglement networks. In extending the arguments of the Glass-Rubber model for semicrystalline polymers, we assume the sum of the crystallinity parameter (a measure equivalent to degree of crystallinity) for both phases must sum up to 1 i.e. $v_{a}+v_{\beta}=1$. The complete constitutive formulation for deviatoric Cauchy stress, $\mathbf{S}$ is an additive response of the weighted contributions of the two processes to the bond-stretching and the conformational deviatoric Cauchy stresses. Therefore, the total twoprocess Cauchy stress becomes:

$$
\boldsymbol{\sigma}=\mathbf{S}+\boldsymbol{\sigma}_{m} \quad \text { where } \quad \mathbf{S}=v_{\alpha} \mathbf{S}_{\alpha}^{b}+v_{\beta} \mathbf{S}_{\beta}^{b}+\mathbf{S}^{c}
$$

$\mathbf{S}_{\alpha}^{b}$ and $\mathbf{S}_{\beta}^{b}$ represent the alpha- and beta-process dominant deviatoric bond-stretching stress and $\mathbf{S}^{c}$ is the conformational deviatoric stress. The entangled molecular network apply for both crystalline and amorphous phases, hence the $\mathbf{S}^{c}$ has not been weighted between the two phases. In the following sections, the exact formulations for $\mathbf{S}^{b}$ and $\mathbf{S}^{c}$ Cauchy stress components are presented.

\subsubsection{Bond stretching deviatoric stress components, $\mathbf{S}^{b}$}

With respect to the bond-stretching deviatoric stress component, $\mathbf{S}^{b}$ the total rate of deformation can be additively decomposed into elastic and viscous parts.

$$
\overline{\mathbf{D}}=\overline{\mathbf{D}}^{e}+\overline{\mathbf{D}}^{v}
$$

where $\overline{\mathbf{D}}$ the rate of deformation, has been additively decomposed into elastic, e and viscous, $\mathrm{v}$ parts and an overbar indicates the isochoric contribution. These two parts originate from the elastic bond-stretching, $\overline{\mathbf{D}}^{e}$ and viscous flow of molecular segments, $\overline{\mathbf{D}}^{v}$. The rate of deformation can be modelled by linear elasticity and associative flow rule for the elastic and viscous parts respectively. Therefore the total deviatoric rate of deformation becomes:

$$
\overline{\mathbf{D}}=\frac{\hat{\mathbf{S}}_{j}^{b}}{2 G_{j}^{b}}+\frac{\mathbf{S}_{j}^{b}}{\mu_{j}}=\frac{1}{2 G_{j}^{b}}\left\{\hat{\mathbf{S}}_{j}^{b}+\frac{\mathbf{S}_{j}^{b}}{\phi_{j}}\right\}
$$

where relaxation time, $\phi_{j}=\mu_{j} / 2 G_{j}^{b}$ is defined in terms of a generalized stress-dependent viscosity, $\mu_{j}$ and bond stretching contribution to shear modulus, $G_{j}^{b}$. Also, $j$ refers to either alpha- or beta-process, and $\hat{\mathbf{S}}_{j}^{b}$ is an objective rate of the bond-stretching deviatoric stress, $\mathbf{S}_{j}^{b}$. The Jaumann objective stress rate [19] is adopted here. Therefore, for a given $j$-process, the objective rate of the deviatoric bondstretching Cauchy stress, in the presence of a finite spin, W is expressed as equation (3), where $\dot{S}_{j}^{b}$ is the rate of change of bond-stretching deviatoric component Cauchy stress.

$$
\hat{\mathbf{S}}_{j}^{b}=\dot{\mathbf{S}}_{j}^{b}-\mathbf{W} \mathbf{S}_{j}^{b}+\mathbf{S}_{j}^{b} \mathbf{W}
$$

For numerical predictions to fit experimental data accurately, each of the alpha- and beta-processes require a spectrum of relaxations times, $\phi_{j}$ reflecting the range of 
molecular packing densities, and hence, activation barriers, where flow events occur in the polymer. However, it has been shown by Wu and Buckley [20] that in the region of yield and post-yield polymer deformation, the full relaxation spectrum collapses into a single relaxation time such that each alpha- or beta-process corresponds to a geometric mean relaxation time i.e. $\phi_{\alpha}$ and $\phi_{\beta}$.

The relaxation time varies with: (a) temperature $T$, (b) structure of the material as expressed through Tool's fictive temperature, $T_{f}$ (c) isotropic invariants of stress: mean stress, $\sigma_{m}$ and octahedral shear stress, $\tau_{\text {oct }}$ [15]. The mean relaxation time, $\phi_{j}$ can be related to its value, $\phi_{j, 0}^{*}$ in a stress-free reference configuration. The relaxation time for $j$-th process in terms of structure, $a_{S, j}$ temperature, $a_{T, j}$ and stress, $a_{\sigma, j}$ shift factors is:

$$
\phi_{j}=a_{T, j} a_{S, j} a_{\sigma, j} \phi_{j, 0}^{*}
$$

The stress-free reference value for the relaxation time spectrum $\phi_{j, 0}^{*}$ is the linear viscoelastic limit of the geometric mean relaxation time of the spectrum. In the following, the formulations for each of the above shift factors are presented.

Temperature Shift Factor: Temperature effects on the relaxation time are introduced by the Arrhenius equation, for both phases as:

$$
a_{T, j}=\exp \left[\frac{\Delta H_{0, j}}{R}\left(\frac{1}{T}-\frac{1}{T^{*}}\right)\right]
$$

where $\Delta H_{0, j}$ represents the enthalpic contribution to the activation energy barrier associated with the $j$-phase which can be either alpha- and beta-segments and $T^{*}$ is a reference temperature.

Stress Shift Factor: In this paper, it was assumed that structural relaxation occurs both in the amorphous and pseudo-amorphous phases. This agrees with observation of a broadened glass transition zone made by Struik [21]. Structural relaxation is implemented for both the alphaand beta-phases through Tool's fictive temperature. The structural shift factor, $a_{S, j}$ for the $j$-phase becomes:

$$
a_{S, j}=\exp \left[\frac{C}{T_{f, j}-T_{\infty}}-\frac{C}{T_{f}^{*}-T_{\infty}}\right]
$$

where $C=$ Cohen-Turnbull constant, $T_{f, j}$ is fictive temperature for the $j$-phase, and $T_{f}^{*}$ is corresponding reference fictive temperature for a case where the structure is always in equilibrium, $T_{\infty}$ is Vogel temperature (where relaxation time, $\left.\phi_{j} \rightarrow \infty\right)$. The structural relaxation evolves with plastic strain: an empirical formulation for the dependence of fictive temperature on time and viscous rate of deformation has been proposed [22]. The same implementation was used here for the two processes.

Stress Shift Factor: This result from the combined effects of the mean stress, $\sigma_{m}$ and the bond-stretching octahedral shear stress, $\tau_{o c t, j}^{b}$ of the deforming polymer for each of the $j$-segments i.e. alpha- and beta- of the polymer. Using Eyring rate kinetics, the stress shift factor for the $j$-process,
Table 1. Flow parameters for 3 grades of polypropylene at temperature, $T=25^{\circ} \mathrm{C}$. For details of the 3 grade of Polypropylene, please refer to [12].

\begin{tabular}{|c|c|c|c|c|}
\hline \multirow{2}{*}{ Material } & $V_{s, \alpha}$ & $V_{s, \beta}$ & $\phi_{0, \alpha}^{*}$ & $\phi_{0, \beta}^{*}$ \\
\cline { 2 - 5 } & \multicolumn{2}{|c|}{$\left[\times 10^{-3} \mathrm{~m}^{3} / \mathrm{mol}\right]$} & \multicolumn{2}{|c|}{$\mathrm{sec}$} \\
\hline ICIW PP & 3.9273 & 0.2956 & 92.21 & $1.852 \times 10^{-4}$ \\
\hline PLW PP & 4.1076 & 0.53227 & 93.54 & $1.014 \times 10^{-4}$ \\
\hline PLB PP & 4.1732 & 0.70634 & 73.37 & $0.822 \times 10^{-4}$ \\
\hline
\end{tabular}

where $j=\alpha, \beta$, can be expressed in terms of the shear and pressure activated stress factor as [20]:

$$
a_{\sigma, j}=\frac{V_{s} \tau_{o c t, j}^{b}}{2 R T} \frac{\exp \left\{-\frac{V_{p} \sigma_{m}}{R T}\right\}}{\sinh \left\{\frac{V_{s} \tau_{o c t, j}^{b}}{2 R T}\right\}}
$$

where $V_{s, j}$ and $V_{p, j}$ are shear- and pressure-activation volumes respectively, for each of the $j$-process, $R=$ gas constant, and $T=$ current temperature.

\subsubsection{Conformational deviatoric stress component, $\mathbf{S}^{c}$}

The conformational deviatoric stress, $\mathbf{S}^{c}$ originates from the entropy elastic stress of entanglements/networks of the polymer. It is convenient to calculate the $\mathbf{S}^{c}$ directly by differentiating the conformational free energy density, $A^{c}$ thus:

$$
\mathbf{S}^{c}=\bar{\lambda}_{i} \sum_{i}^{3} \frac{\partial A^{c}}{\partial \bar{\lambda}_{i}} \mathbf{u}_{i} \otimes \mathbf{u}_{i}-p \mathbf{I}
$$

where $p=\frac{1}{3} \operatorname{trace}\left[\frac{\partial A^{c}}{\partial \bar{\lambda}_{i}} \mathrm{u}_{i} \otimes \mathrm{u}_{i}\right], \bar{\lambda}_{i}(i=1, \ldots, 3)$ is the eigenvalues of the left Cauchy-Green tensor, $\overline{\mathbf{B}}$ while $\mathrm{u}_{i}$ is the unit eigenvectors of $\overline{\mathbf{B}}$, and $p$ is an unknown hydrostatic pressure. The definition of the conformational entropy free energy function, $A^{c}$ is derived from the physically based function proposed by Edwards and Vilgis [23] for a network of cross-linked and entangled freely jointed chains of finite length regarded as chains of 'Kuhn' segments.

\subsection{Model predictions}

The constitutive mathematics developed above were implemented into a MATLAB script and used initially to predict the response of polypropylene. Also, an ABAQUS user-defined material sub-routine was created. A few model predictions were investigated and results validated against compression data for three polypropylene grades obtained across a wide range of strain rates [12]. The model parameters were adjusted to experimental data and Table 1 shows the activation volumes and reference relaxation times obtained for the two viscoelastic activation processes. 


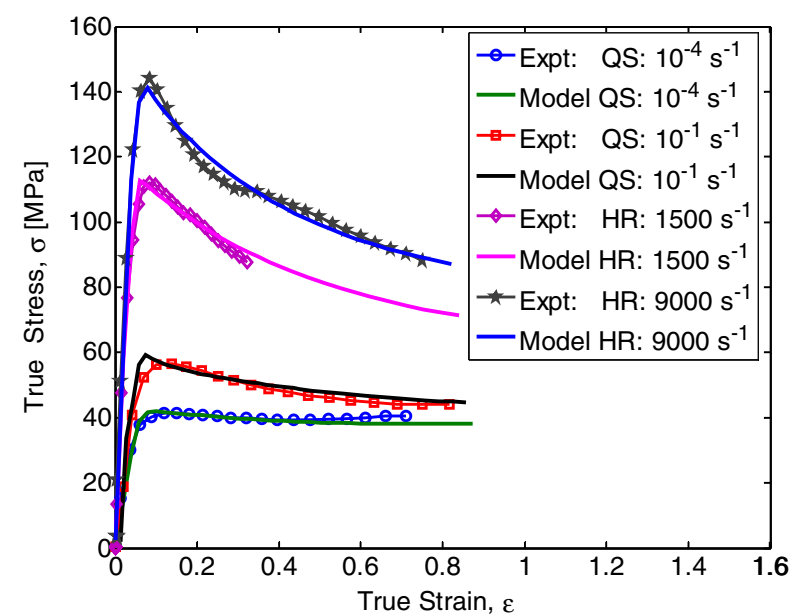

Fig. 2. Comparison between model predictions and experiment for compression tests under varying strain rate for reference grade polypropylene (ICIW) at test temperature, $T=25^{\circ} \mathrm{C}$.

\subsubsection{Rate-dependent response}

Simulations of compression tests were carried out for quasi-static $\dot{\varepsilon}=10^{-4} \mathrm{~s}^{-1}$ and $\left.10^{-1} \mathrm{~s}^{-1}\right)$ and high $(\dot{\varepsilon}=$ $1500 \mathrm{~s}^{-1}$ and $9000 \mathrm{~s}^{-1}$ ) strain rates. Comparisons were made with experimental data of the reference polypropylene (ICIW) grade and this is shown in Fig. 2. Generally, the model predictions agree quite well with experimental data for linear viscoelastic, yield and post-yield constitutive responses. However, the experiment shows a diffused or broadened yield region at quasi-static rates which has not been captured accurately by the model. The disparity is attributed to the choice of a single-mode relaxation time. It has been shown by Wu and Buckley, that in order to fit data accurately around yield, a spectrum of relaxation times is required [20].

\subsubsection{Temperature-dependent response}

Tensile tests on the PLB polypropylene grade at quasistatic rates, $\dot{\varepsilon}=10^{-3} \mathrm{~s}^{-1}$ under varying temperatures were compared with model predictions, as shown in Fig 3. Temperature-data at high rates were not available. The experiments showed a brittle response at temperatures around room temperature, i.e. fracture before yield. At temperatures around the melting temperature of polypropylene $\left(T_{m}=165^{\circ} \mathrm{C}\right)$, a ductile response was observed. Comparison between the model and experiments agree well, qualitatively and quantitatively.

\subsubsection{Effect of de-ageing}

Structural relaxation in semicrystalline polymers has been assumed in this paper to occur not only in the amorphous phase (through the beta-process viscoelastic relaxation), but also through relaxation of the pseudo-amorphous phase (by the alpha-process viscoelastic relaxation). In order to assess the validity of this assumption, two simulations chosen from quasi-static and high strain rates were carried

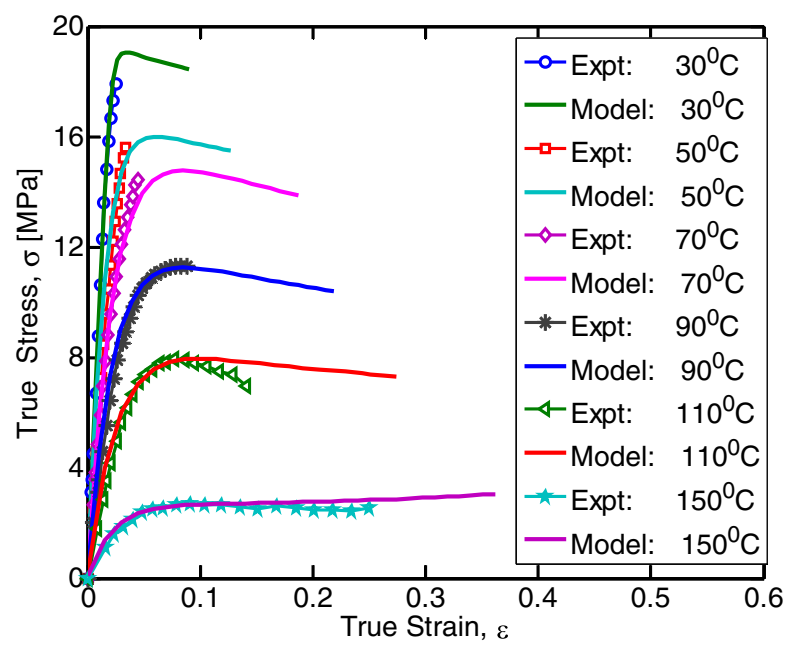

Fig. 3. Comparison between model predictions and experiment for tensile tests at strain rate, $\dot{\varepsilon}=10^{-3} \mathrm{~s}^{-1}$ under varying temperatures, for blended (PLB) polypropylene grade.

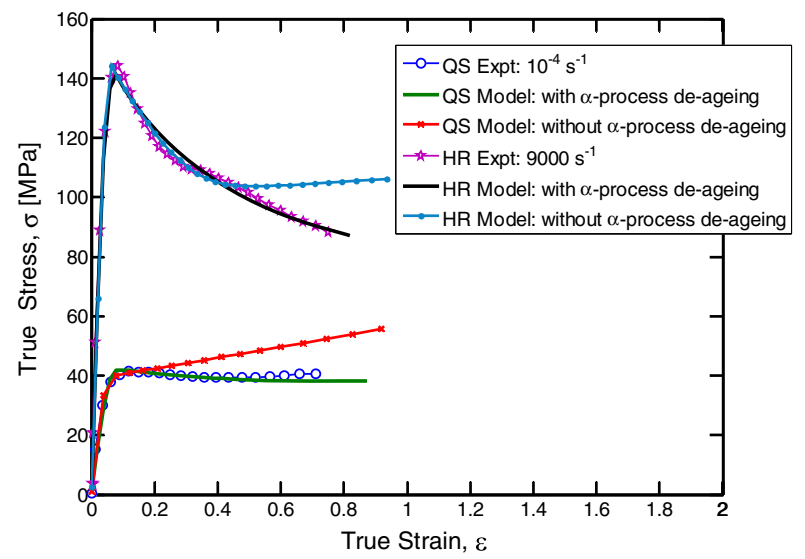

Fig. 4. Comparison of compression test data and model predictions at quasi-static (QS) and high (HR) loading rates on reference polypropylene grade at test temperature $25^{\circ} \mathrm{C}$.

out and results compared with experimental data as shown in Fig 4. In the first simulation, only the amorphous phase was allowed to undergo structural evolution through Tool's fictive temperature. A second simulation was carried out where both alpha- and beta-processes were allowed to undergo structural change (i.e. de-age) with plastic deformation. Model predictions based on incorporating or excluding the de-ageing of the alpha-process were compared with experiments, as shown in Fig. 4. The results showed a good agreement between experiment and model predictions in the case where de-ageing of the alphaprocess was allowed. This validates the assumption that the structural evolution of semicrystalline polymers is not restricted solely to the amorphous phases but as well as the constrained non-crystalline phase.

\subsubsection{Simulating nonlinear compressive yield response}

The objectives here were to determine the formulation for the rate-dependent compressive yield response and then compare the model predictions with experiment. Based on 


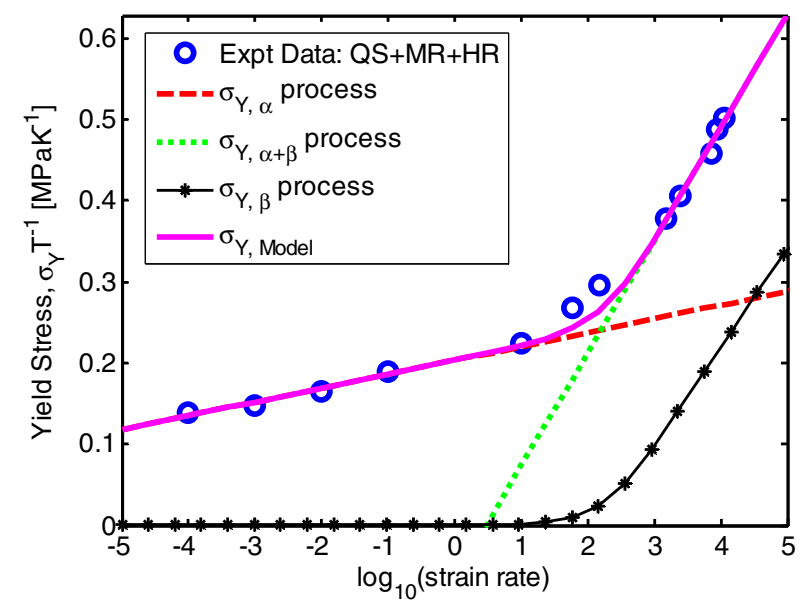

Fig. 5. Prediction of rate-dependent compressive yield behaviour of reference polypropylene (ICIW) grade at test temperature of $T=25^{\circ} \mathrm{C}$. The predicted nonlinear Eyring function, $\sigma_{\text {Model }}$ was based on the two-process model.

the constitutive mathematics of the two process model, the compressive yield stress can be determined by adopting the arguments of two-process Ree-Eyring rate kinetics.

Let us define the following indices of the Ree-Eyring kinetics as: (i) Activation index, $B_{j}=2 \frac{V_{p, j}}{V_{s, j}}$; (ii) Relaxation index, $A_{j}=\frac{2 \sqrt{2} a_{S, j} a_{T, j} G_{j}^{b} \phi_{0, j}^{*}}{\tau_{0, j}}$; (iii) mean stress index, $\gamma_{j}=$ $\exp \left\{\frac{-\sigma_{m} V_{p, j}}{R T}\right\}$; (iv) a pressure-activated stress factor, $\sigma_{0, j}=$ $\frac{R T}{V_{p, j}}$; and (v) shear-activated stress factor, $\tau_{0, j}=\frac{2 R T}{V_{s, j}}$.

The compressive yield response follows two dominant mechanical relaxations according to the two-process model i.e. Processes 1 and 2. Process 1 mechanical response is dominant at low strain rate or high temperature test conditions while Process 2 becomes significant beyond a critical strain rate, $\dot{\varepsilon}_{\text {crit }}$ and yield stress, $\sigma_{\text {crit }}$. At any stage in the deformation, the two processes act cooperatively to describe the compressive yield response of the semicrystalline polymer leading to a total stress, $\sigma_{\text {Total }}$.

The formulations for these processes are given thus:

$$
\begin{gathered}
\left.\sigma_{y, j}\right|_{1}=\frac{3 v_{j} \sigma_{0, j} B_{j}}{\sqrt{2}-B_{j}}\left\{\ln A_{j}+\ln |\dot{\varepsilon}|\right\}, \\
\left.\sigma_{y, j}\right|_{2}=\frac{3 v_{j} \sigma_{0, j} B_{j}}{\sqrt{2}} \ln \left\{\frac{\gamma_{j} A_{j}}{2} \dot{\varepsilon}+\sqrt{\left(\frac{\gamma_{j} A_{j}}{2} \dot{\varepsilon}\right)^{2}+1}\right\},
\end{gathered}
$$

and total stress becomes:

$$
\left.\sigma_{y, j}\right|_{\text {Total }}=\left.v_{\alpha} \sigma_{y, j}\right|_{1}+\left.v_{\beta} \sigma_{y, j}\right|_{2}+\sigma_{m} .
$$

The comparison between experiment and model predictions of the strain rate dependence of material yield behaviour of PP using the proposed model based on data of Fig. 2 is illustrated Fig. 5. This shows the interaction of the two processes and how their co-operative effects lead to the total stress.

Eyring plots of the two polypropylene grades are shown in Fig. 6.

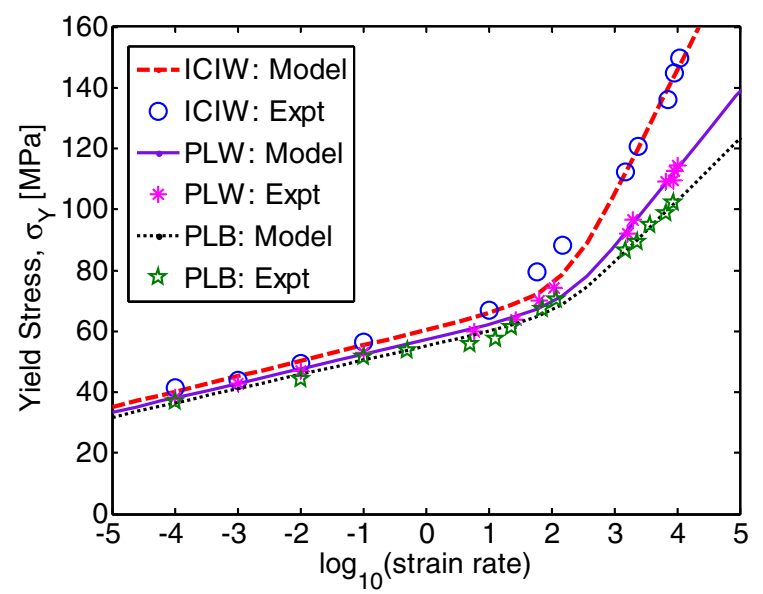

Fig. 6. Comparison of Eyring plots for experimental and model predictions for ICIW and PLB polypropylene grades all tested at $T=25^{\circ} \mathrm{C}$.

\section{Conclusions}

The constitutive behaviour of semicrystalline polymers has not previously been modelled across a wide range of strain-rates extending up to impact rates. This paper presents a three-dimensional physically-based constitutive model for polypropylene, based on the well-known physical framework of rate-dependent deformation proposed by Bauwens which established a correlation between the observed transition in flow stress of a material and the secondary beta-transition of viscoelastic behaviour. It was assumed that the overall stress experienced by the semicrystalline polymer results from a parallel contribution of an elasto-viscoplastic stress in the amorphous, crystalline and pseudo-zones of the microstructure, with the contributing stresses weighted by a crystallinity parameter. Also, the model has been validated against compression tests carried out on two grades of PP tested across eight decades of time. Model predictions were found to agree quite well with the linear viscoelastic, rate-dependent yield and postyield strain softening of polypropylene.

\section{References}

1. K. Hong, A. Rastogi, G. Strobl, Macromolecules 37, 10165 (2004).

2. Y. Wang, E. M. Arruda, Journal of Engineering Materials and Technology, Transactions of the ASME 128, 551 (2006).

3. J. Sweeney, R. Spares, M. Woodhead, Polymer Engineering and Science 49, 1902 (2009).

4. C. Ebert, W. Hufenbach, A. Langkamp, M. Gude, Polymer Testing 30, 183 (2011).

5. M. Zrida et al., Polymer Testing 29, 685 (2010).

6. R. Bouix, P. Viot, J.-L. Lataillade, International Journal of Impact Engineering 36, 329 (2009).

7. M. Xu, L. Wang, Mechanics of Materials 38, 68 (2006).

8. A. D. Mulliken, M. C. Boyce, International Journal of Solids and Structures 43, 1331 (2006).

9. S. C. Chou, K. D. Robertson, J. H. Rainey, Experimental Mechanics 13, 422 (1973). 
10. S. M. Walley, J. E. Field, P. H. Pope, DYMAT Journal 1, 211 (1994).

11. T. Gómez-del Río, J. Rodríguez, European Polymer Journal 46, 1244 (2010).

12. M. I. Okereke, C. P. Buckley, C. R. Siviour, Mechanics of Time-Dependent Materials, DOI: 10.1007/s11043012-9167-z, 1 (2012).

13. T. Ree, H. Eyring, in Rheology: Theory and Applications, F. R. Eirich, Ed. (Academic Press, New York, 1958), vol. 2, pp. 83-142.

14. J. C. Bauwens, Journal of Materials Science 7, 577 (1972).

15. C. P. Buckley, D. C. Jones, Polymer 36, 3301 (1995).

16. C. P. Buckley, D. C. Jones, D. P. Jones, Polymer 37, 2403 (1996).
17. P. J. Dooling, C. P. Buckley, S. Rostami, N. Zahlan, Polymer 43, 2451 (2002).

18. C. P. Buckley, P. J. Dooling, J. Harding, C. Ruiz, Journal of the Mechanics and Physics of Solids 52, 2355 (2004).

19. G. A. Holzapfel, Nonlinear Solid Mechanics: A Continuum Approach for Engineering. (John Wiley and Sons Inc, London, UK, 2000), vol. 1.

20. J. J. Wu, C. P. Buckley, Journal of Polymer Science, Part B: Polymer Physics 42, 2027 (2004).

21. L. C. E. Struik, Polymer 30, 815 (1989).

22. A. C. Y. Lew, C. P. Buckley, in 23rd annual meeting of the polymer processing society, Salvador, Brazil. (2007), vol. 4, Pages, pp. 2294-2298.

23. S. F. Edwards, T. Vilgis, Polymer 27, 483 (1986). 